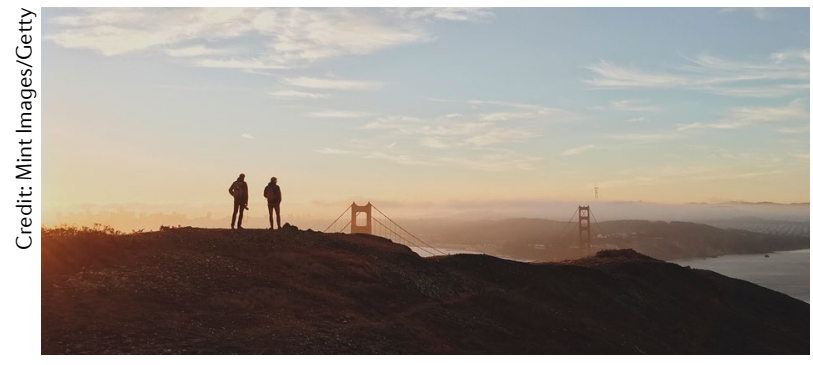

NAVIGATION

\section{A position on vision}

Vision has a key role in navigation, but does spatial positioning affect the processing of visual information? In a new study, Saleem et al. show that, in mice, location-related activity can be detected early in visual processing, in the primary visual cortex (V1), suggesting that navigation influences vision.

The authors recorded calcium signals in excitatory cells in V1 while the mice explored a virtual reality corridor by running on a treadmill. This corridor comprised two identical sections; each section included two different landmarks, so along the corridor, each landmark occurred twice and thus two repeating scenes were generated.

Predictably, some of the $\mathrm{V} 1$ neurons responded to the repeated scenes in a similar fashion. However, many V1 neurons showed differential responses to the repeated scenes, suggesting that the animal's position in the corridor influences V1 activity.

The authors next examined whether the differences in V1 responses across sections were related to location-related activity in the hippocampus - a key region involved in navigation - so they allowed mice to explore the corridor while they simultaneously recorded from V1 and hippocampal area CA1 using electrode arrays. Notably, to determine an animal's subjective position in the environment, the authors trained mice to lick for water when the animals had reached a specific region in the corridor (the reward zone).

As above, many V1 neurons showed stronger activity in one of the two sections of the corridor, and, predictably, CA1 neurons were preferentially activated in a given position in the corridor (their 'place field'). Indeed, the position of the animal on the track could be decoded from the activity of either V1 or CA1 neurons using an independent Bayesian decoder. Thus, neurons in both brain regions encode location-specific signals.

Despite the training, animals made some errors by licking before or after the reward zone, and the authors found that there were correlated errors in activity in CA1 or V1. Interestingly, Bayesian decoding of licking-related activity in CA1 or V1, regardless of whether the licking occurred in the reward zone, placed the animals in this area. This suggests that the activity in V1 and CA1 encodes the subjective location of the animal.

This study reveals that early visual activity is influenced by signals for spatial location that are represented in the hippocampus. Moreover, these signals relate to where the animal perceives itself to be, as opposed to its actual location.

Darran Yates

ORIGINAL ARTICLE Saleem, A. B. et al. Coherent encoding of subjective spatial position in visual cortex and hippocampus. Nature https://doi.org/10.1038/ s41586-018-0516-1 (2018)

\title{
Number crunching
}

Humans are the only animals to have developed the ability to represent numerical value with symbols. This capacity is thought to be built upon more basic quantification skills that allow us to accurately estimate the size (numerosity) of a set of objects. However, we know little about the coding of numerical value in the human brain. Kutter et al. have now used single cell recording methods to demonstrate the existence of distinct populations of 'number neurons' representing either symbolic numbers or numerosity in the human medial temporal lobe (MTL).

The MTL is part of a wider 'number network' identified in human and non-human primate studies. To examine the contributions of individual MTL neurons to number encoding, the authors recorded the activity of 585 neurons from the MTL (including neurons in the amygdala, parahippocampal cortex, entorhinal cortex and hippocampus) of 9 subjects undergoing neurosurgery. During recording, the subjects participated in a task in which they were shown numerical values presented in a symbolic format (Arabic numerals) or a nonsymbolic format (random dot arrays).

The authors found that $16 \%$ of the recorded neurons were tuned to numerosity: they demonstrated peak activity when the subject viewed a dot array corresponding to one particular numerical value. Their responses to the presentation of other numerical values decreased progressively according to the size of the difference between the presented value and their preferred value, a property known as a numerical distance effect. $3 \%$ of the recorded neurons responded specifically to numerical values presented as symbols. These neurons exhibited a smaller numerical distance effect, suggesting a sharper tuning to numerical value. Only a few neurons were tuned to both nonsymbolic and symbolic numerical values.

To examine the encoding of numerical value at a population level, the authors trained a machine classifier to recognize numerical values on the basis of the firing of the population of selective neurons. The classifier was capable of decoding numerical information from a novel set of firing data at above chance levels for both symbolic and nonsymbolic representations, indicating that MTL population activity can accurately encode numerical representations.

These findings reveal the existence of two mostly separate populations of 'number neurons' representing symbolic and nonsymbolic numerical value in the human MTL. Further analysis of the functional properties of these neurons and their place in the number network may provide clues to the mechanisms underlying our unique symbolic numerical abilities. Katherine Whalley

ORIGINAL ARTICLE Kutter, E. F. et al. Single neurons in the human brain encode numbers. Neuron https://doi.org/10.1016/j.neuron.2018.08.036 (2018) FURTHER READING Nieder, A. The neuronal code for number. Nat. Rev. Neurosci. 17, 366-382 (2016)

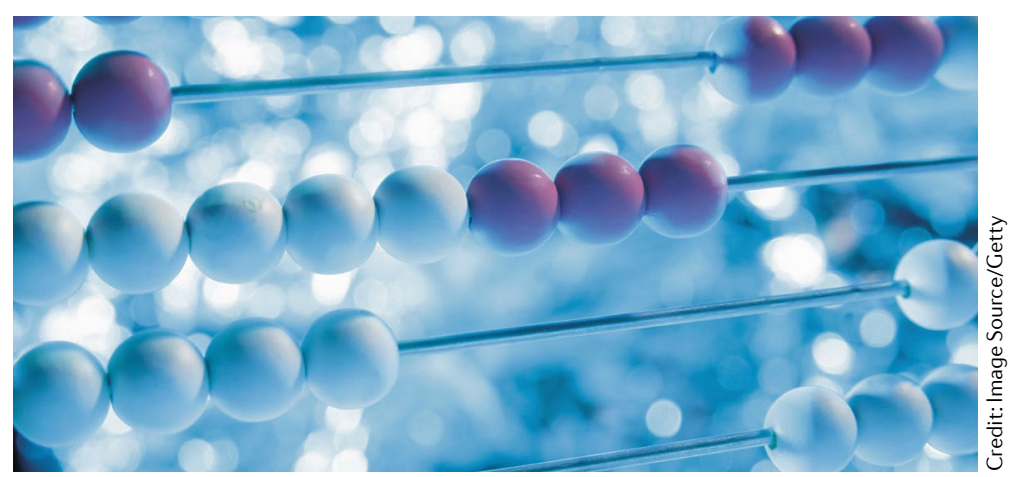

